\title{
Tragfähigkeitsuntersuchungen an Verbundträgern aus BS-Holz und Faserverbundkunststoff-Lamellen
}

\author{
H.J. Blaß, M. Romani
}

Das Ziel der Untersuchung war, Angaben zum Trag- und Verformungsverhalten von Verbundträgern aus BS-Holz und Faserverbundkunststoffen zu erhalten. Dazu wurde der Einfluss des Verstärkungsgrades, der geometrischen Anordnung der Verstärkung und der Einfluss verschiedener Verstärkungslamellen genauer untersucht und verstärkte BS-Holz Träger unter praxisnahen Bedingungen hergestellt.

Investigations of the load carrying behaviour of composite glued laminated timber beams reinforced with fibre reinforced plastic

The aim of the investigation was to study the load carrying and deformation behaviour of composite glued laminated timber beams reinforced with fibre reinforced plastic (FRP). For this purpose the influence of amount and position of the reinforcement and of different FRP laminations and adhesives was determined. Reinforced beams were produced under practice conditions.

\section{Einleitung ${ }^{1}$}

Die Tragfähigkeit des BS-Holzes wird durch die mechanischen Eigenschaften des Holzes und durch die Festigkeit der Keilzinkenverbindungen begrenzt. So versagen BS-Holz-Träger unter einer Momentenbeanspruchung hauptsächlich im Zugbereich in der Nähe von Wuchsunregelmäßigkeiten oder an den Keilzinkenverbindungen (KZV). Steigerungen der Tragfähigkeit und Biegesteifigkeit von BS-Holz können

H. J. Blaß, M. Romani

Universität Karlsruhe (TH)

Lehrstuhl für Ingenieurholzbau und Baukonstruktionen

Ernst Gaber-Straße

Geb. 10.50, 76128 Karlsruhe

1 Die hier dargestellten Versuche wurden in den Jahren 1998 bis 2000 im Auftrag der DGfH durchgeführt und aus Haushaltsmitteln des Bundesministeriums für Wirtschaft und Technologie (BMWi) über die Arbeitsgemeinschaft industrieller Forschungsvereinigungen „Otto von Guerike“ e.V. (AiF) gefördert. 
durch Verstärkungen mit Werkstoffen hoher Festigkeit und hoher Steifigkeit erzielt werden. Dies sind z. B. Laminate aus Kunststofffasern wie Aramid oder Kohlenstoff.

Neben sehr guten Festigkeits- und Steifigkeitseigenschaften bieten Faserverbundkunststoffe (FVK) weitere Vorteile. Durch ihr geringes Gewicht bei hoher Tragfähigkeit wurden sie zunächst in der Luft- und Raumfahrttechnik eingesetzt. Seit einigen Jahren werden diese Werkstoffe auch in der Automobilindustrie und im Bauwesen verwendet. Im Massivbau werden Faserverbundkunststoffe neben der hohen Zugfestigkeit auch aufgrund ihrer Beständigkeit gegenüber korrosiven Medien eingesetzt. Anwendung finden die FVK-Lamellen auch im Bereich der nachträglichen Verstärkung von Tragwerken, da aufgrund ihrer einfachen Applikation ein Austausch von Bauteilen häufig vermieden werden kann.

Für eine gezielte Verwendung von FVK-Lamellen zur Verstärkung von BS-Holz Trägern im Ingenieurholzbau spricht die Möglichkeit, den Baustoff BS-Holz besser als bisher ausnutzen zu können. Der Verstärkungseffekt erfolgt durch die Entlastung der in der Zugzone befindlichen Schwachstellen. Weiterhin wird durch die Traglaststeigerung eine höhere Ausnutzung des plastischen Potentials von BS-Holz erzielt. Aufgrund des Verstärkungseffektes können Bretter der Sortierklasse S7 oder MS7, die bisher nicht für die Herstellung von BS-Holz verwendet wurden, wirtschaftlich eingesetzt werden.

Die genannten Vorteile ermöglichen kleinere Querschnitte bei gleicher Traglast. Hieraus ergeben sich günstigere Transport- und Einbaubedingungen und ein geringeres Gewicht der Tragkonstruktion. Im Bereich der Sanierung und Nutzungsänderung von Tragwerken ist aufgrund der einfachen Applikation der FVK-Lamellen eine nachträgliche Verstärkung wirtschaftlich möglich. Um das Trag- und Verformungsverhalten dieser Bauteile zu untersuchen, wurden an der Versuchsanstalt für Stahl, Holz und Steine, Abt. Ingenieurholzbau der Universität Karlsruhe (TH) Versuche an 38 verstärkten Trägern durchgeführt.

\section{Literaturübersicht}

In der Vergangenheit wurden verschiedene Möglichkeiten untersucht, Biegeträger aus Holz mit anderen Materialien im Zug- und Druckbereich zu verstärken. Als Verstärkung wurden Stahl z.B. von Peterson (1965), Lantos (1970), Bulleit et al. (1989) oder Lamellen aus glasfaserverstärkten Kunststoffen (GFK) z.B. von Biblis (1965) und Hernandez et al. (1997) verwendet.

Bei Stahl steht das hohe Gewicht, seine Korrosionsanfälligkeit, das ungünstige Verhalten im Brandfall und die im Vergleich zu Holz geringere elastische Dehnung einer breiten Verwendung als Verbundwerkstoff mit BS-Holz im Wege. Mit glasfaserverstärkten Lamellen müssen hohe Verstärkungsgrade gewählt werden, um signifikante Steigerungen der Tragfähigkeit und Biegesteifigkeit zu erzielen. Dies liegt an dem im Vergleich zu Holz nur etwa dreifach höheren Elastizitätsmodul der Glasfaserlamellen.

Rowlands et al. (1986) führten neben umfangreichen Versuchen an verschiedenen Klebstoff - FVK Kombinationen auch Versuche an BS-Holz Trägern 
mit Verstärkungen aus GFK-Lamellen durch. In den Versuchen wurde eine Steigerung der Biegesteifigkeit und der Traglast durch die GFK-Lamellen Verstärkung festgestellt.

Ehlbeck et al. (1987) untersuchten Verbundträger aus BS-Holz mit Glasfaserverbundprofilen. Die Glasfaserverbundprofile waren beidseitig mit Deckschichten aus Kiefer-Messerfurnieren versehen, um eine bessere Verklebbarkeit zu erreichen. Ziel der Untersuchung war es, die Tragfähigkeit von mit Glasfaser-Verbund-Profilen verstärkten BS-Holz Biegeträgern zu untersuchen und gleichzeitig die Tragfähigkeitssteigerung gegenüber unverstärkten BS-Holz Trägern zu ermitteln. Ergebnis der Untersuchung war, dass Holzbereiche mit Ästen stärker entlastet wurden, als die Bereiche, bei denen Keilzinkenverbindungen vorhanden waren.

Van de Kuilen (1991) untersuchte mit GFK-Profilen verstärkte Vollholzquerschnitte, die sowohl im Zugbereich als auch im Zug- und Druckbereich mit zwei unterschiedlichen Verstärkungsgraden hergestellt wurden. Ergebnis dieser Untersuchung war, dass die Biegesteifigkeit bedeutend erhöht werden konnte und diese mit der elastischen Verbundtheorie zutreffend berechnet werden kann.

Plevris et al. (1992) untersuchten Verbundquerschnitte aus Holz und kohlefaserverstärkten Kunststoffen (CFK) und entwickelten Berechnungsgleichungen für kombinierte Belastungen aus Normalkraft und Biegemoment für verschiedene Versagensarten. Es wurde festgestellt, dass auch kleine Verstärkungsgrade zu einer signifikanten Verstärkung führen.

Hernandez et al. (1997) führten eine Untersuchung an BS-Holz Biegeträgern mit Verstärkungen aus GFK-Lamellen mit je einer Verstärkungslage in der Zug- und Druckzone und Versuche mit zwei Verstärkungslagen in der Zugzone durch. Die in der Druck- und Zugzone verstärkten Träger wiesen eine höhere Steifigkeit und eine geringere Tragfähigkeit auf als die mit zwei Lagen in der Zugzone verstärkten Träger.

Neben einer Steigerung der Tragfähigkeit wurde bei den meisten Untersuchungen eine reduzierte Streuung der Versuchswerte festgestellt. Bei den meisten Untersuchungen wurde, wie auch im Rahmen der hier dargestellten Untersuchung von Blaß et al. (2000), das Erreichen der Biegezugfestigkeit, mit einem anschließend beobachteten Schubversagen, ausgehend vom Anriss, beobachtet.

\section{Versuchsmaterial}

\subsection{Brettmaterial}

Für die Bauteilversuche wurde Holz aus maschinell sortierten Brettern der Sortierklassen MS7, MS10 und MS17 ausgewählt. Bei der Auswahl von Brettern der Sortierklasse MS10 wurden die Bretter mit den niedrigsten Elastizitätsmoduln und Rohdichten des jeweils vorhandenen Brettangebotes ausgewählt. Dies geschah, um einen möglicht großen Unterschied in den Eigenschaften des Holzes im Verhältnis zu den Eigenschaften der verwendeten FVK-Lamellen $\mathrm{zu}$ erhalten und die 
Verwendbarkeit von Brettern minderer Qualität für die Herstellung von BS-Holz auf zu zeigen. Für alle Versuche wurde das Sortiment Fichte/Tanne verwendet.

Der Grundaufbau aller Versuchsträger bestand aus Brettern der Sortierklasse MS10, die zur Ober- bzw. Unterkante des Querschnitte immer geringere Elastizitätsmoduln und Rohdichten aufwiesen.

\subsection{Faser-Verbund-Kunststoff Lamellen (FVK-Lamellen)}

Für die Untersuchungen standen zunächst vier verschiedene FVK-Lamellen zur Verfügung, von denen drei für die Bauteilversuche verwendet wurden. Drei der vier FVK-Lamellen bestanden aus Kohlenstofffasern (CF) und eine aus Aramidfasern (AF). In Tabelle 1 und Tabelle 2 werden die Bezeichnungen und Eigenschaften der FVK-Lamellen entsprechend den Herstellerangaben und aus Zugversuchen beschrieben. Die AFK-Lamelle L3 war werksseitig auf beiden Seiten, die CFKLamellen L1, L2 und L4 werksseitig auf einer Seite angeschliffen.

Tabelle 1: Vorhandene FVK-Lamellen

\begin{tabular}{|c|c|c|c|}
\hline Abk. & Produktbezeichnung & Hersteller / Vertrieb & Typ \\
\hline L1 & Sika CarboDur S & Sika Chemie GmbH & CFK \\
\hline L2 & Sika CarboDur H & Sika Chemie GmbH & CFK \\
\hline L3 & - & Akzo Nobel Faser AG & AFK \\
\hline L4 & S\&P CFK Lamelle & Ispo GmbH & CFK \\
\hline
\end{tabular}

Für die Bauteilversuche wurden die zwei CFK-Lamellen L1, L4 und die AFK-Lamelle L3 verwendet.

Tabelle 2: Materialangaben der FVK-Lamellen

\begin{tabular}{|c|c|c|c|c|}
\hline Abk. & $\begin{array}{c}\text { Zug-E-Modul }^{1)} \\
\text { Mittelwert } \\
{\left[\mathrm{N} / \mathrm{mm}^{2}\right]}\end{array}$ & $\begin{array}{c}\text { Zugfestigkeit }^{1)} \\
\text { Mittelwert } \\
{\left[\mathrm{N} / \mathrm{mm}^{2}\right]}\end{array}$ & Dicke & Breite \\
{$[\mathrm{mm}]$} & {$[\mathrm{mm}]$} \\
\hline L1 & 173.000 & 3.050 & 1,2 & 100 \\
\hline L2 & 304.000 & 1.680 & 1,4 & 50 \\
\hline L3 & 74.000 & 995 & 1,8 & 132 \\
\hline L4 & 199.000 & 2.570 & 1,4 & 100 \\
\hline
\end{tabular}

1) aus Zugversuchen an $50 \mathrm{~mm}$ breiten Proben, Versuchsanzahl 5

\subsection{Klebstoffe}

Als Klebstoffe standen zwei Epoxidharzklebstoffe, die vom Hersteller für eine Verwendung mit den Lamellen L1 und L2 bzw. L4 vorgesehen sind und zwei Klebstoffe aus der BS-Holz Herstellung zur Verfügung. In Tabelle 3 sind die Bezeichnungen dieser Klebstoffe dargestellt.

Die Epoxidharzklebstoffe K1 und K2 wurden aufgrund der bewährten Verklebung von CFK-Lamellen im Massivbau ausgewählt. Der Resorcinharzklebstoff K4 wurde 
gewählt, da eine Verklebung mit Resorcinharzklebstoffen mit der AFK-Lamelle L3 möglich erschien, und dieser Klebstoff bei BS-Holz Herstellern Verwendung findet. Der Polyurethanklebstoff K3 wurde in das Versuchsprogramm aufgenommen, da dieser Klebstoff sowohl bei BS-Holz Herstellern Verwendung findet und gleichzeitig, neben der Verklebung der AFK-Lamelle L3, eine Verklebung mit CFK-Lamellen aufgrund von Vorversuchen aussichtsreich erschien.

Tabelle 3: Vorhandene Klebstoffe

\begin{tabular}{|c|c|c|}
\hline Abk. & Produktbezeichnung & Hersteller / Vertrieb \\
\hline K1 & Sikadur-30 & Sika Chemie GmbH \\
\hline K2 & ispo Concretin SK 41 & ispo GmbH \\
\hline K3 & Collano Purbond HB 110 & Ebnöther AG \\
\hline K4 & $\begin{array}{r}\text { Dynosol S-199 } \\
\text { mit Härter H-629 }\end{array}$ & Dyno Industries A.S. \\
\hline
\end{tabular}

Aufgrund der Voruntersuchung wurden für die Bauteilversuche mit einer Herstellung im Labor der Klebstoff K2 und bei der praxisnahen Herstellung bei einem BS-Holz Hersteller der Klebstoff K3 verwendet. Der Epoxidharzklebstoff K2 wurde für die Laborherstellung ausgewählt, da eine tragfähige Verklebbarkeit zwischen den Fügeteilpartnern Holz und FVK erwartet wurde und die Verarbeitbarkeit des Klebstoffes K2 aufgrund eines geringeren Füllstoffanteiles einfacher war als beim Klebstoff $\mathrm{K} 1$.

\section{Bauteilversuche}

\subsection{Aufbau, Herstellung und Prüfung der Versuchsträger}

Da die Prüfkörper der Versuchsreihen Tr-1 und Tr-2 mit Brettlamellen ohne KZV hergestellt werden sollten, wurde für alle Träger eine Spannweite von $4,20 \mathrm{~m}$ bei einer Gesamtlänge von 4,50 m aufgrund der beschränkten Länge der Einzelbretter festgelegt. In Tabelle 4 sind die durchgeführten Versuchsreihen dargestellt. Als Ausgangsmaterial für die Bauteilversuche wurde für alle Versuchsreihen MS10 und für die Versuchsreihen Tr-1 und Tr-2 im Randbereich bei den äußersten zwei Holzlamellen auch MS7 verwendet. Bei den Versuchsreihen Tr-5 und Tr-6 wurden im Randbereich der Zugzone MS17 Brettlamellen angeordnet. In Tabelle 5 ist der mittlere Längsschwingungs-E-Modul und die mittlere Rohdichte für den Randbereich und den Gesamtquerschnitt angegeben. Bei den Versuchsreihen Tr-3 bis Tr-8 wurden die Brettlamellen mit KZV bei den jeweils gleichzeitig hergestellten Versuchsreihen (Tr-3/Tr-4; Tr-5/Tr-6 und Tr-7/Tr-8) aus jeweils der Bretthälfte zweier Bretter hergestellt, deren übrige Bretthälften in einem Vergleichsträger der jeweils anderen Versuchsreihe an gleicher Stelle eingebaut wurden. Der Aufbau der Versuchsträger ist in Bild 1 dargestellt. 


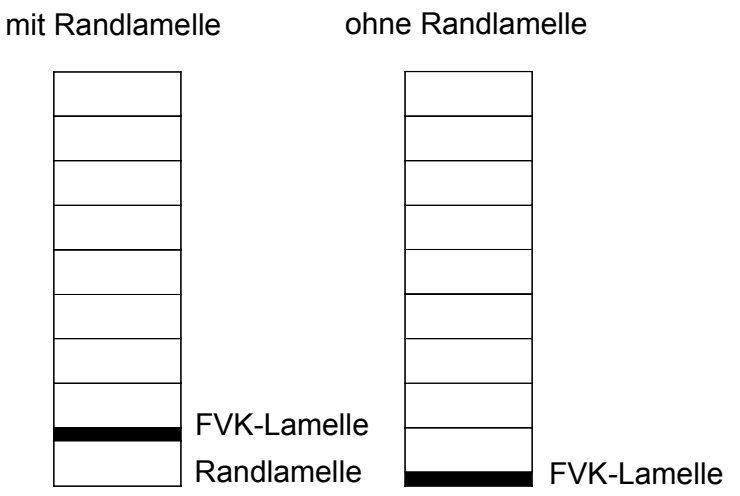

Bild 1: Bezeichnungen der Querschnittsteile und Aufbau der Versuchsträger

Tabelle 4: Versuchsprogramm der Bauteilversuche

\begin{tabular}{|c|c|c|c|c|c|c|c|}
\hline Reihe & $\begin{array}{c}\text { Anzahl } \\
\text { der } \\
\text { Versuche }\end{array}$ & $\begin{array}{c}\text { Sortierklasse } \\
\text { der } \\
\text { Einzelbretter }\end{array}$ & $\begin{array}{c}\text { Randlamelle } \\
\text { aus } \\
\text { Holz }\end{array}$ & $\begin{array}{c}\text { Mittlere } \\
\text { Höhe/Breite } \\
{[\mathrm{mm}]}\end{array}$ & $\begin{array}{c}\text { FVK- } \\
\text { Lamelle } \\
\text { (Anzahl) }\end{array}$ & $\begin{array}{c}\text { Kleb- } \\
\text { stoff }\end{array}$ & KZV \\
\hline Tr-1 & 5 & MS7 / MS10 & MS7 / MS10 & $308 / 100$ & L1 (1) & K2 & ohne \\
\hline Tr-2 & 5 & MS7 / MS10 & MS7 / MS10 & $312 / 100$ & L4 (2) & K2 & ohne \\
\hline Tr-3 & 5 & MS10 & MS10 & $308 / 100$ & L1 (1) & K2 & mit \\
\hline Tr-4 & 5 & MS10 & MS10 & $312 / 100$ & L4 (2) & K2 & mit \\
\hline Tr-5 & 5 & MS10/ MS17 & MS17 & $312 / 100$ & L3 (4) & K3 & mit \\
\hline Tr-6 & 5 & MS10 / MS17 & MS10 & $312 / 100$ & L3 (4) & K3 & mit \\
\hline Tr-7 & 5 & MS10 & - & $308 / 100$ & L1 (1) & K3 & mit \\
\hline Tr-8 & 3 & MS10 & - & $310 / 100$ & L4 (2) & K3 & mit \\
\hline
\end{tabular}

Aufgrund der geringen Ausbeute an MS7 wurden MS7 Bretter nur bei den ersten beiden Versuchsreihen im Randbereich der Bauteile verwendet. Bei den übrigen Versuchsreihen wurde auf MS7 Brettlamellen verzichtet und dafür MS10 Brettlamellen mit möglichst niedrigem Elastizitätsmodul und niedriger Rohdichte im Randbereich angeordnet. Das verwendete Brettangebot hatte mit einem mittleren Längsschwingungs-E-Modul von $9800 \mathrm{~N} / \mathrm{mm}^{2}$ einen vergleichsweise geringen Elastizitätsmodul für MS10 Bretter. Die Keilzinkenverbindungen wurden versetzt im Bereich zwischen den Lasteintragungspunkten angeordnet. Hierbei wurden die oberen zwei und die unteren drei Brettlamellen mit einer Keilzinkenverbindung versehen. Die FVK-Lamellen wurden über die gesamte Länge und Breite der Träger angeordnet. 
Tabelle 5: Längsschwingungs-E-Modul und Rohdichte bei der jeweiligen Feuchte der Einzelbretter

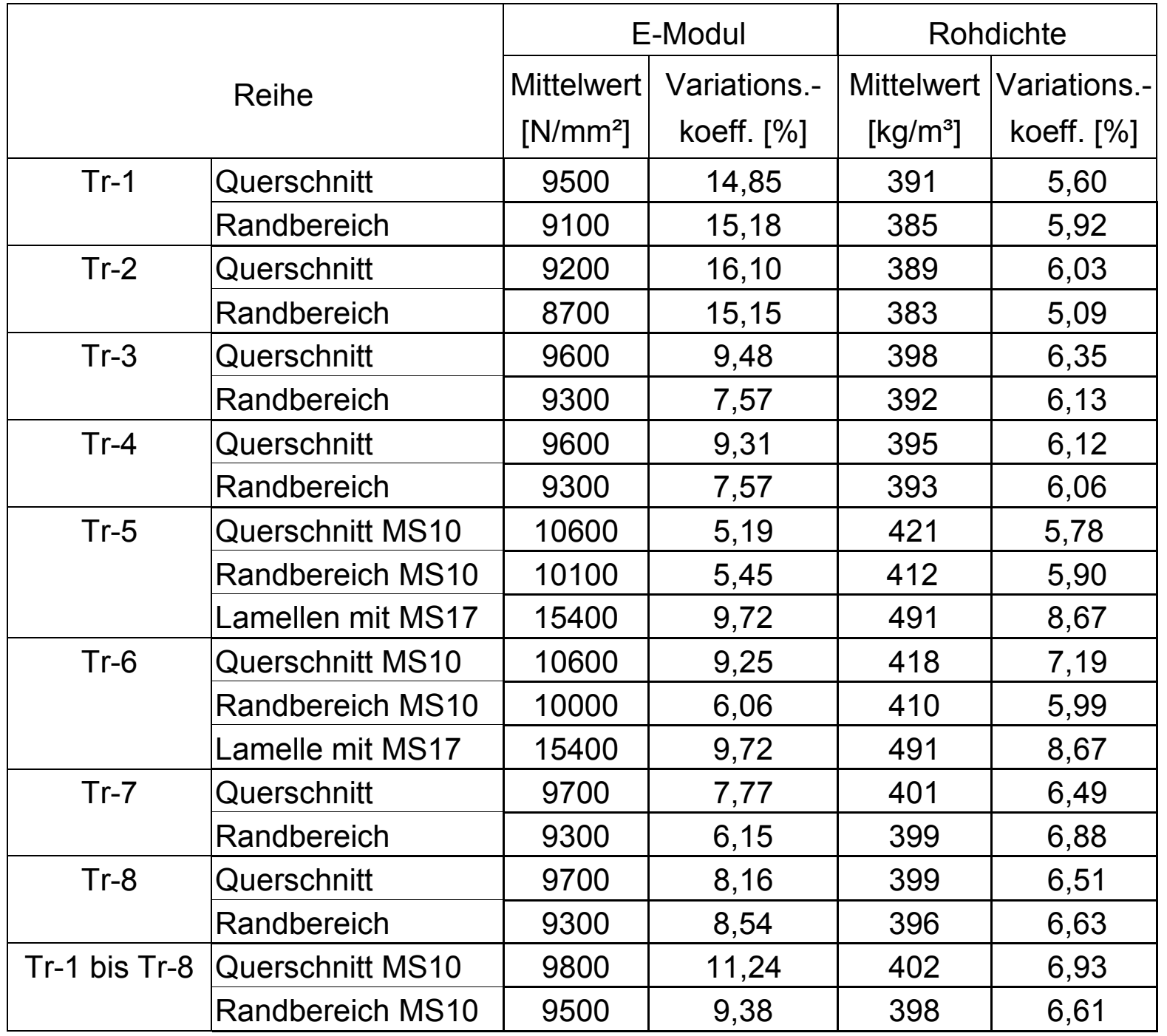

Randbereich: Zwei obersten Holzlamellen und drei untersten Holzlamellen Querschnitt: Alle Holzlamellen des Querschnittes

Die Versuchsreihen Tr-1 bis Tr-4 mit dem Klebstoff K2 wurden im Labor hergestellt. Der Epoxidharzklebstoff wurde bei diesen Versuchsreihen zur Verklebung zwischen den CFK-Lamellen und zwischen CFK-Lamellen und Holz verwendet. Das Sollmaß der Klebefugendicke mit dem Epoxidharzklebstoff betrug $1 \mathrm{~mm}$. Für die Versuchsreihen Tr-1 bis Tr-4 wurden die Grundträger beim BS-Holz Hersteller vorgefertigt. Die Verstärkungslagen und die Randlamellen wurden im Prüflabor bei Normalklima 20/65 aufgeklebt. Die Verklebung der CFK-Lamellen erfolgte nach dem Anschleifen und Entfetten der jeweiligen Oberfläche der CFKLamelle. Die Randlamellen wurden am Tag zuvor bzw. am gleichen Tag auf ihr Sollmaß gehobelt.

Die Versuchsreihen Tr-5 bis Tr-8 wurden als komplette Verbundträger bei einem BS-Holz Hersteller hergestellt, bei dem der Polyurethanklebstoff K3 in der normalen Herstellung von BS-Holz verwendet wird. Beim Klebstoffauftrag auf die FVK-Lamelle 
wurde abweichend von der üblichen BS-Holz Herstellung eine Trägerlamelle aus Holz verwendet, auf welcher die FVK-Lamelle lose aufgelegt wurde.

Die Bauteilversuche wurden in Anlehnung an DIN EN 408 durchgeführt. Die Verformungsgeschwindigkeit betrug zwischen 15 und $20 \mathrm{~mm} / \mathrm{min}$. Die Versuche wurden an einer 400 kN EPZ-Eigenbau und 100 kN EPZ-Eigenbau durchgeführt. Der Versuchsaufbau ist in Bild 2 dargestellt. Die Verschiebungen wurden in Trägermitte an der Oberkante durch einen induktiven Wegaufnehmer mit einem Nennweg von $200 \mathrm{~mm}$, an den Auflagern beidseitig mit induktiven Wegaufnehmern mit einem Nennweg von $2 \mathrm{~mm}$ sowie im mittleren Bereich mit zwei induktiven Wegaufnehmern mit einem Nennweg von $10 \mathrm{~mm}$, bezogen auf die geometrische Mittellinie des Querschnitts, gemessen. Die Auswertung des Elastizitätsmoduls nach DIN EN 408 erfolgte mit den gemittelten Werten der Wegaufnehmer W10. Die Gesamtverformung bezogen auf die Spannweite von 4,20 m wurde aus der an der Bauteiloberkante gemessenen Vertikalverformung und unter Berücksichtigung der Eindrückung am Auflager ermittelt. Die Eindrückung am jeweiligen Auflager wurde aus dem Mittelwert der dort befindlichen Wegaufnehmer ermittelt.

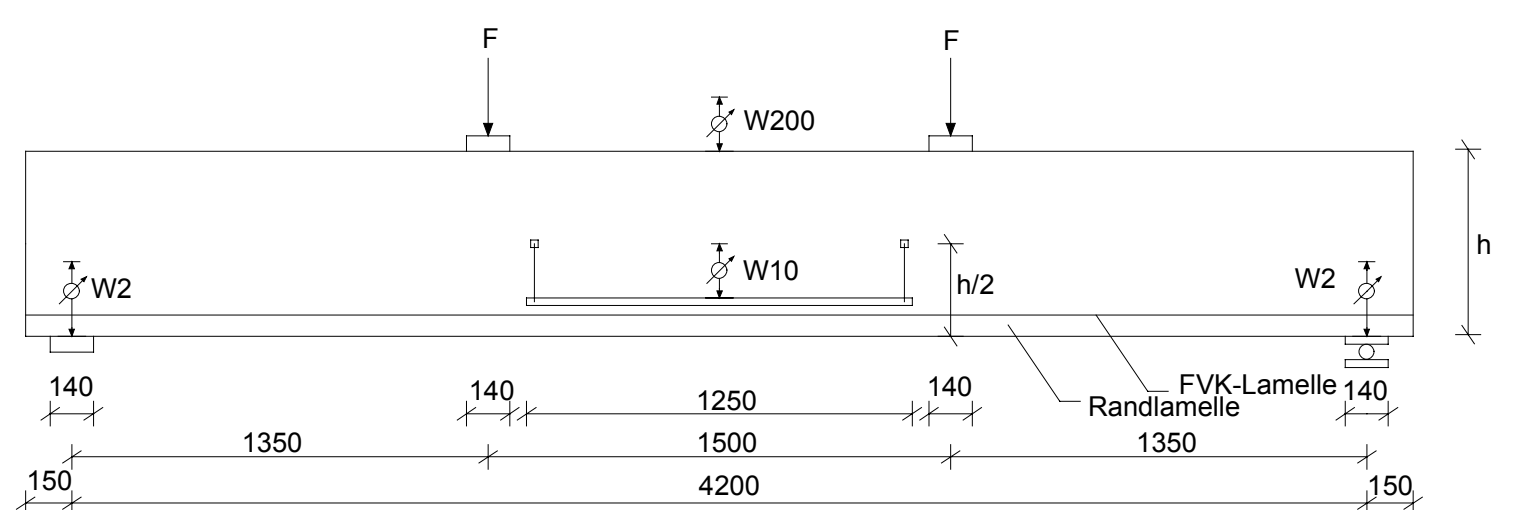

[Maße in $\mathrm{mm}$ ]

Bild 2: Versuchsaufbau der Bauteilversuche

\subsection{Ergebnisse und Vergleich der Versuchsreihen}

\subsubsection{Ergebnisse der Bauteilversuche}

Die Ergebnisse der Versuchsreihen sind in der Tabelle 6 zusammen gefasst. Die Abfolge eines typischen Versagens eines Versuchs mit Randlamelle und ohne Randlamelle zeigt Bild 11. Last-Verformungsdiagramme sind in Bild 7 bis Bild 10 dargestellt. Bild 3 zeigt die Traglasten der Einzelversuche der verschiedenen Versuchsreihen. 
Tabelle 6: Mittelwerte der einzelnen Versuchsreihen

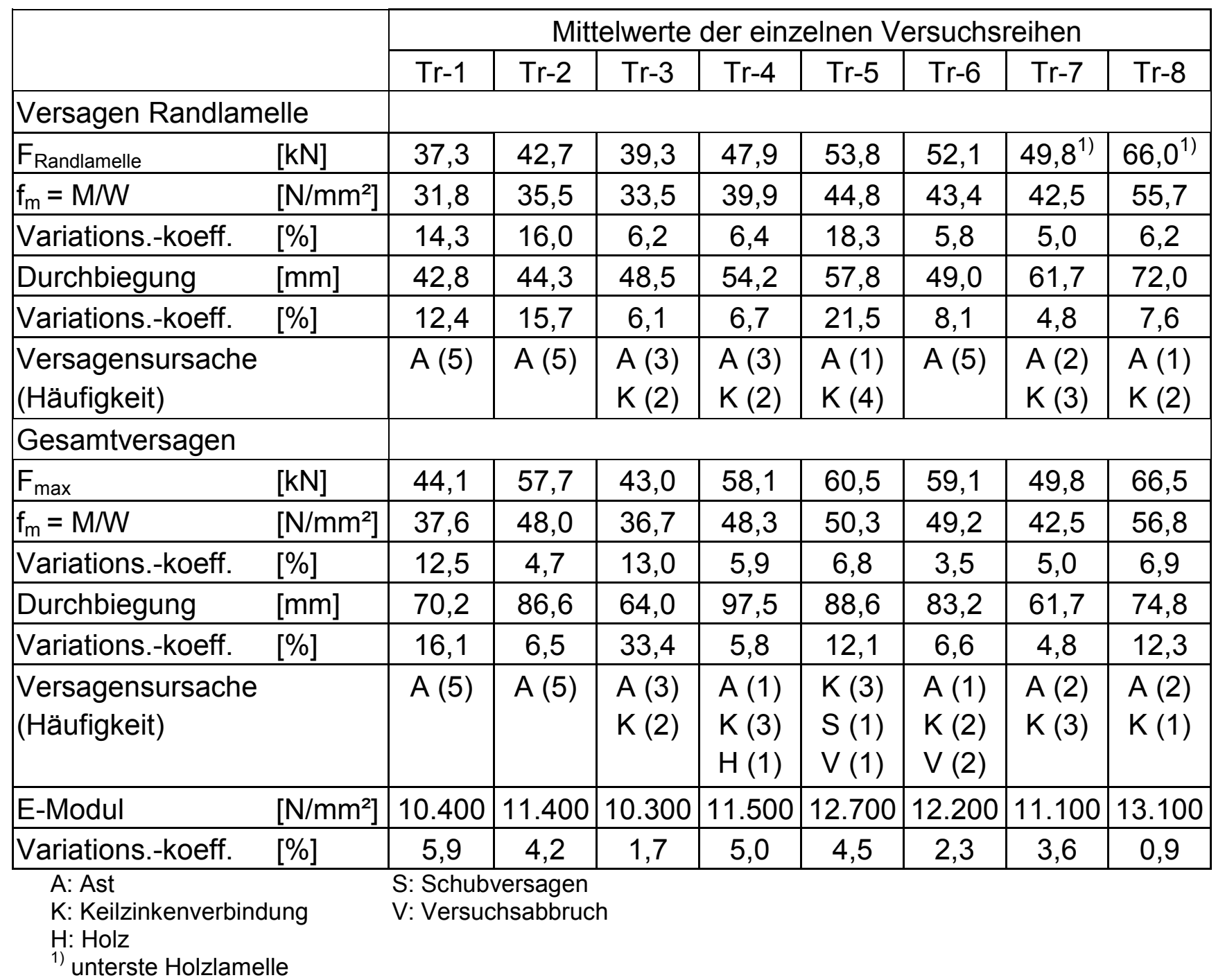

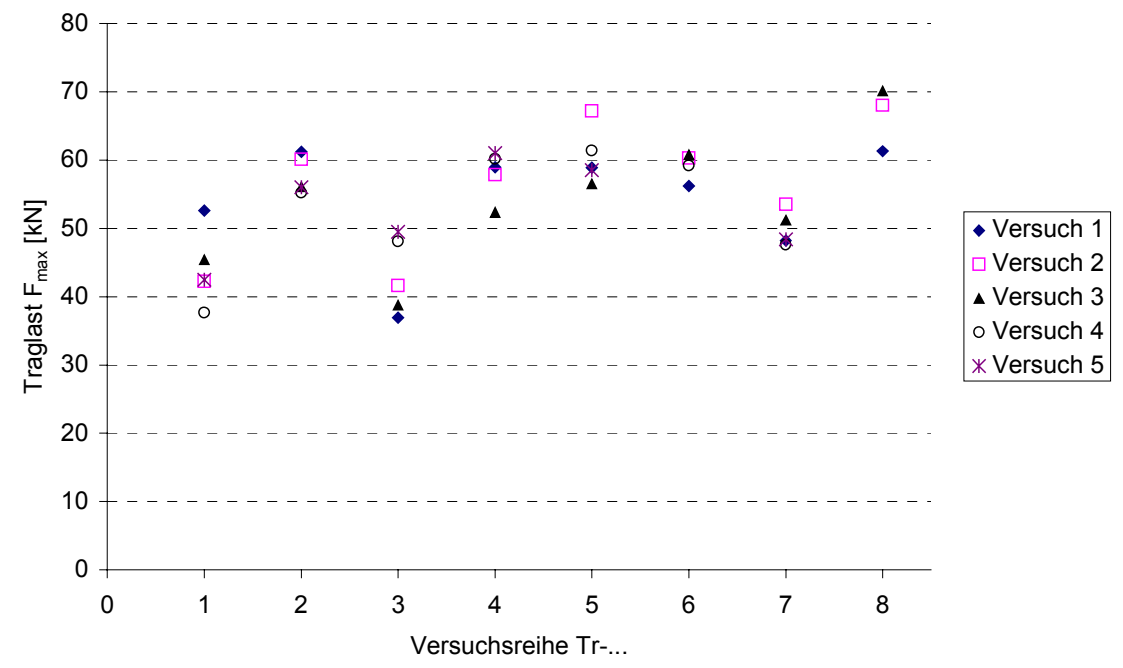

Bild 3: Traglasten $F_{\max }$ der einzelnen Versuche 


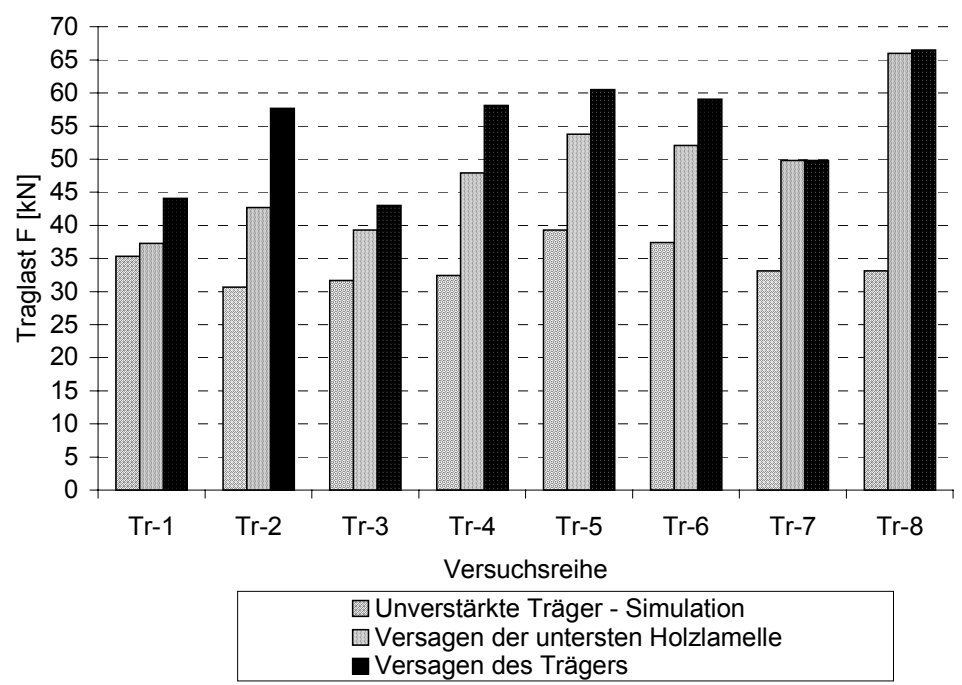

Bild 4: Mittelwerte der Traglast für das Versagen der Randlamelle und des Trägers

In Bild 5 ist ein Vergleich des Elastizitätsmoduls aus den Versuchen und nach einer Berechnung mit der Verbundtheorie für unverstärkte und verstärkte Querschnitte dargestellt. Aus der Berechnung ergibt sich für die verstärkten Querschnitte eine gute Übereinstimmung mit den Versuchsergebnissen. Der tendenziell höhere Elastizitätsmodul aus der Berechung der Träger im Vergleich zu den im Versuch ermittelten Werten, lässt sich durch den höheren Elastizitätsmodul bei dynamisch ermittelten Moduln gegenüber statisch ermittelten Werten der Einzelbretter erklären. Die Eingangswerte dieser Berechnung bestehen aus den Längsschwingungs-E-Moduln, die bei der Holzauswahl für jedes Brett ermittelt wurden. Weiterhin ist zu erkennen, dass eine Erhöhung der Biegesteifigkeit im Vergleich zu unverstärkten Querschnitten vorhanden ist. Aus einem Vergleich der einzelnen Versuchsreihen lässt sich entnehmen, dass ein Einfluss der Lage und Verstärkungsmenge der FVK-Lamelle deutlich erkennbar ist.

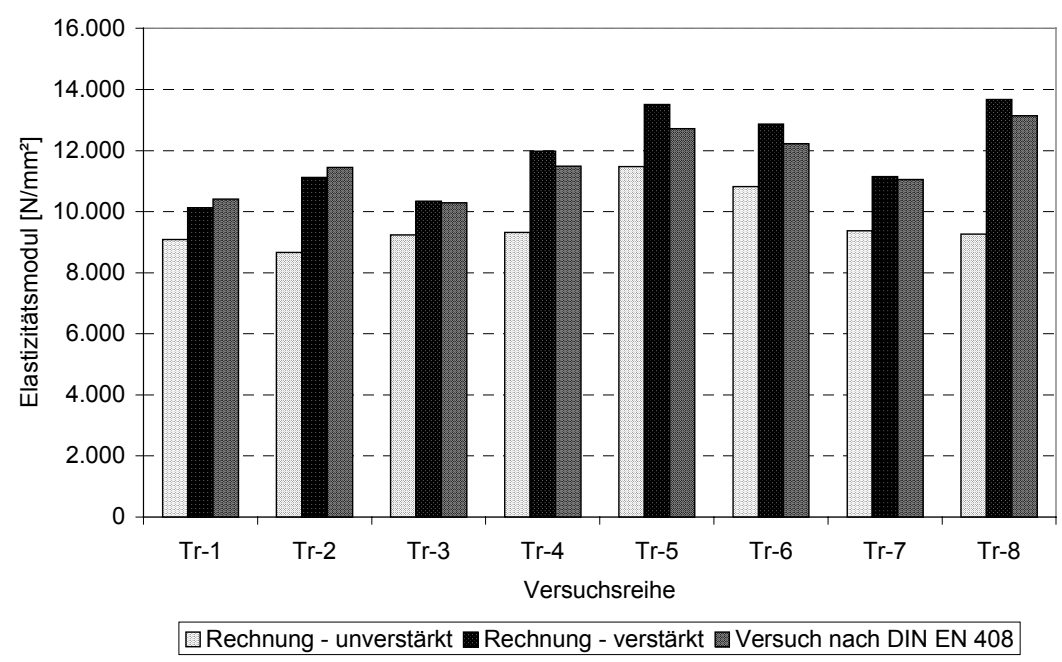

Bild 5: Mittelwerte des Elastizitätsmoduls aus den Versuchen und berechnete Werte des Elastizitätsmoduls für die unverstärkten und verstärkten Querschnitte 
Aus der Erhöhung der Biegesteifigkeit und der Traglast der Verbundträger ergibt sich die Fragestellung, wie groß die Materialeinsparung gegenüber einem unverstärkten Querschnitt ist, der bei gleicher Breite und entsprechend angepasster Querschnittshöhe die gleiche Biegesteifigkeit bzw. Tragfähigkeit hat. In Bild 6 ist diese Materialersparnis bezogen auf den erforderlichen unverstärkten Querschnitt dargestellt. Als Grundlage wurden für die Biegesteifigkeit der unverstärkten Träger die berechneten E-Moduln entsprechend Bild 5 und für die Tragfähigkeit die simulierten Werte nach Bild 4 verwendet.

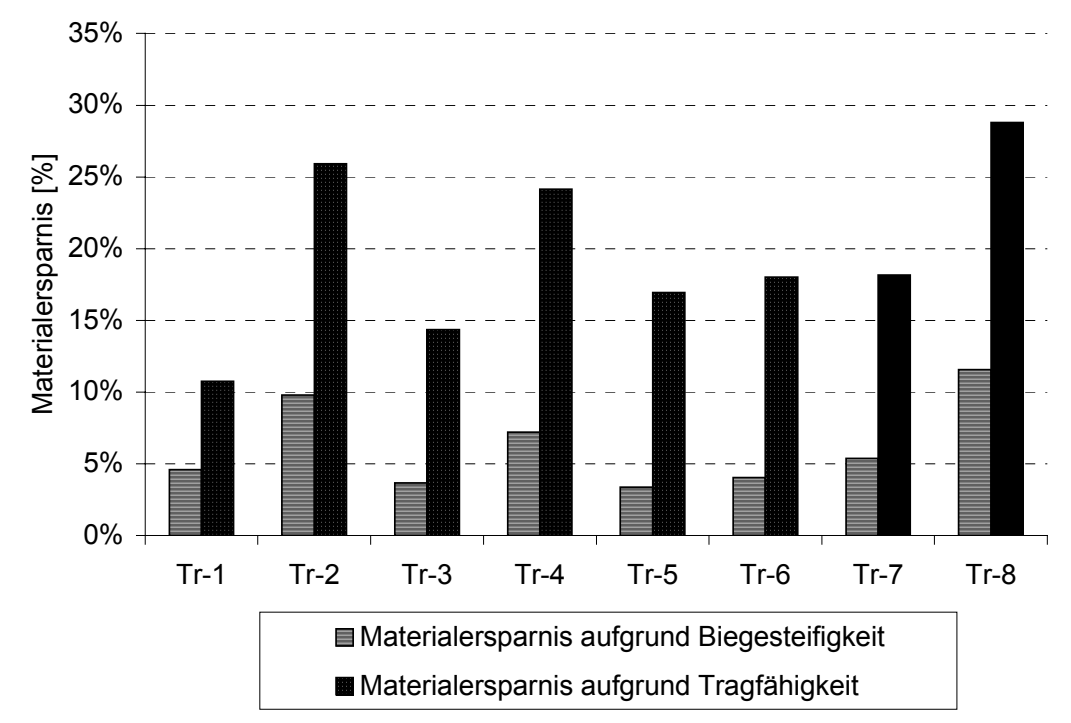

Bild 6: Materialersparnis bei gleicher Querschnittsbreite um die gleiche Biegesteifigkeit bzw. Tragfähigkeit zu erzielen

Wie aus Bild $6 \mathrm{zu}$ erkennen ist, ist die Materialersparnis aufgrund der Tragfähigkeit deutlich höher, als bei Trägern, deren Biegesteifigkeit maßgebend ist.

\subsubsection{Tragverhalten}

Insgesamt traten bei den Bauteilversuchen vier verschiedene Versagensarten auf. Das Tragverhalten war dabei abhängig vom Anteil der Verstärkung am Gesamtquerschnitt, von der Qualität der Klebefuge, der Lage der FVK-Lamelle und den Materialeigenschaften der FVK-Lamelle und des BS-Holzes.

Bei der ersten Versagensart versagten die Bauteile ohne Ankündigung. Für die Versuchsreihen Tr-7 und Tr-8 war dies ein typisches Versagen, da bei diesen Versuchsreihen keine Randlamelle vorhanden war. In Bild 7 ist das Last-Verformungsdiagramm eines solchen Versuches dargestellt. Bei einzelnen Versuchen trat dieses Versagen auch bei Versuchsreihen mit Randlamelle auf. Bei diesen Versuchen war in der Regel nur eine geringe Krümmung der Last-Verformungskurve zu erkennen. 


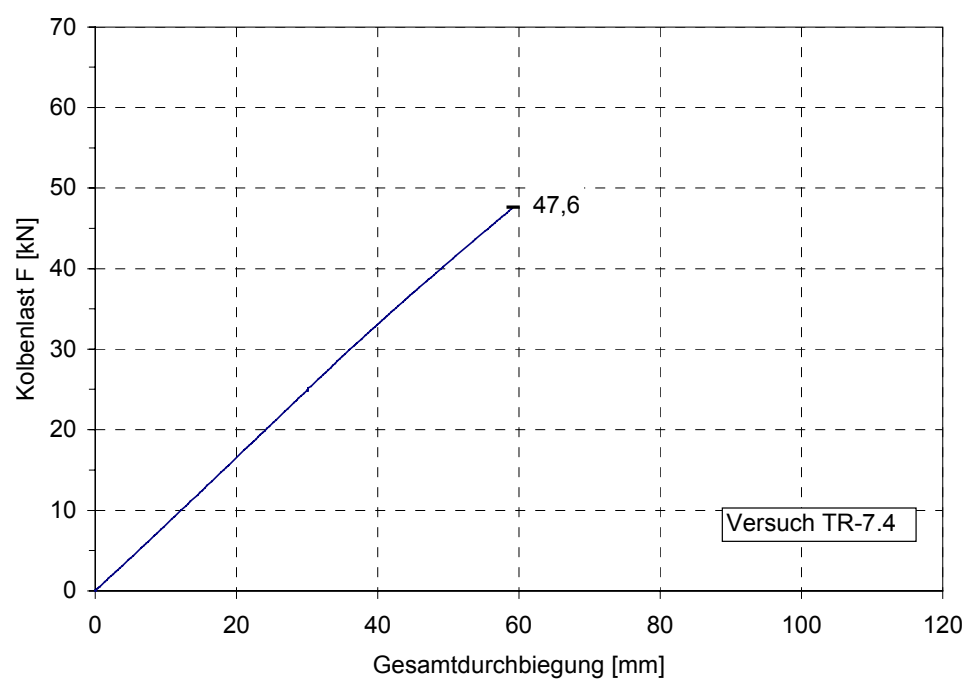

Bild 7: Last-Verformungsdiagramm des Versuchs Tr-7.4

Eine weitere Versagensart war ein Versagen der Randlamelle bei anschließender erneuter Lastaufnahme, ohne die zuvor erreichte Last wieder zu erreichen. In Bild 8 ist das Last-Verformungsdiagramm des Versuchs Tr-3.3 dargestellt, bei dem nach zweimaligem Lastabfall ein Versagen des Gesamtsystems unterhalb der Höchstlast erfolgte.

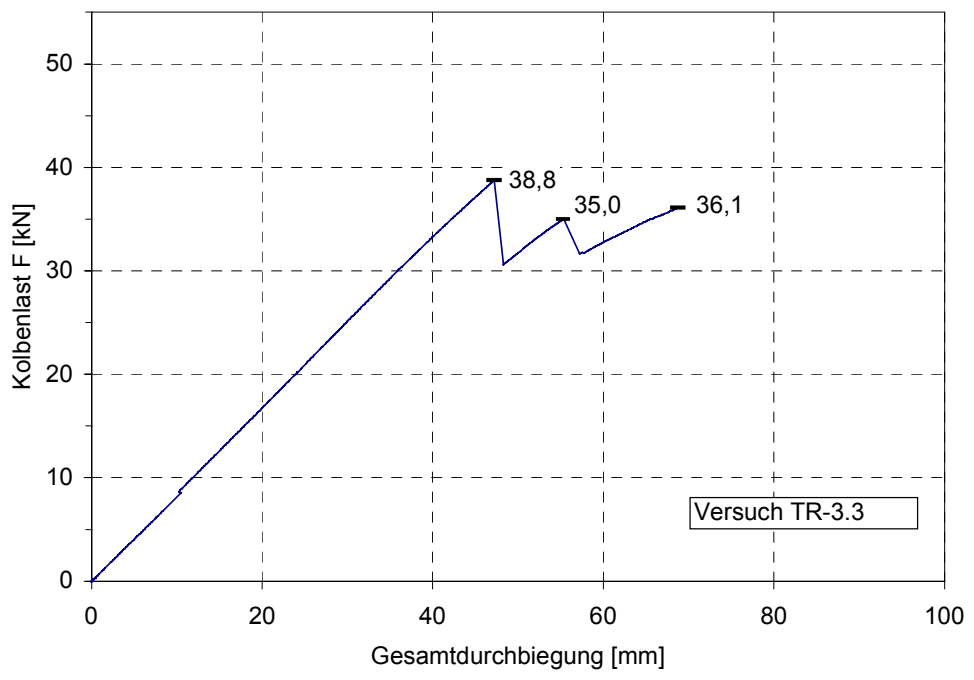

Bild 8: Last-Verformungsdiagramm des Versuchs Tr-3.3

Die dritte Versagensart ist in Bild 9 dargestellt. Nach dem Versagen der Randlamelle kam es zu einer weiteren Steigerung der Last, das Gesamtversagen erfolgte dann durch ein Versagen einer Holzlamelle oberhalb der FVK-Lamelle. 


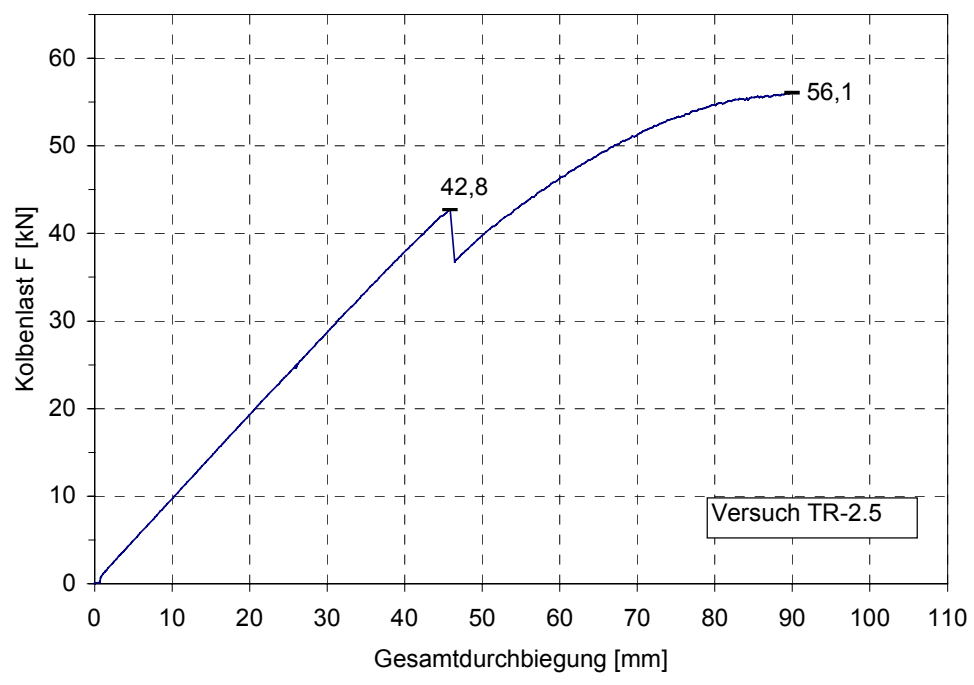

Bild 9: Last-Verformungsdiagramm des Versuchs Tr-2.5

In Bild 10 ist die vierte Versagensart dargestellt, bei der es zu einem stufenweisen Versagen kam. Dieses Versagen trat bei der Versuchsreihe Tr-5 und Tr-6 auf. Bei einigen Versuchen wurden die Versuche ohne ein Gesamtversagen des Bauteils abgebrochen. Nach Rissen in der Randlamelle und der zweiten bzw. dritten Holzlamelle von unten traten sehr große Druckfalten auf. Das Bauteil verformte sich ausgesprochen plastisch. Das Versagen erfolgte in mehreren Stufen, teilweise trat mehrfaches Versagen innerhalb einer Brettlamelle auf. In Bild 12 ist der zum LastVerformungsdiagramm in Bild 10 gehörige Querschnitt bei Versuchsabbruch dargestellt.

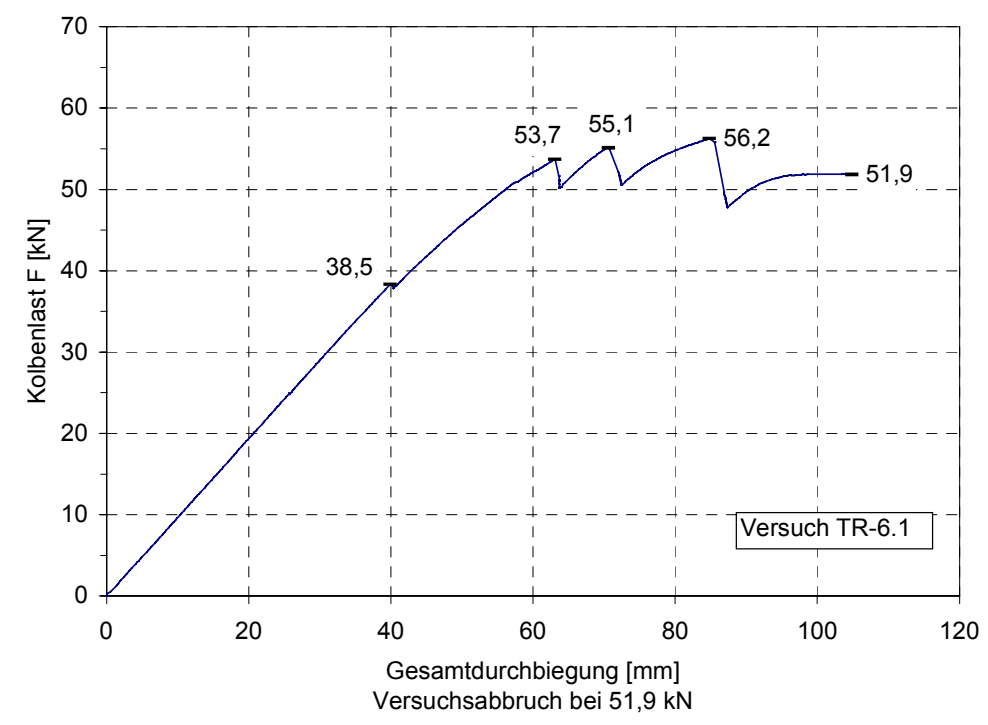

Bild 10: Last-Verformungsdiagramm des Versuchs Tr-6.1

Bis zum ersten Versagen einer Holzlamelle zeigte sich bei den meisten Versuchen keine bzw. nur eine geringe Krümmung der Last-Verformungskurve. Plastische Verformungen traten demnach bei diesen Versuchen nicht oder nur in einem geringem Umfang auf. Trat nach dem ersten Versagen kein Versagen des Gesamtsystems auf, so konnte teilweise ein ausgeprägt plastisches Verhalten beobachtet werden. In Bild 9 ist ein Beispiel mit einem durchschnittlichen plastischen 
Verhalten dargestellt. In einzelnen Versuchen wurden im Querschnitt Druckfalten bis zu etwa 1/3 der Querschnittshöhe beobachtet. Ein Beispiel einer solch stark ausgeprägten Druckfalte ist in Bild 12 dargestellt.

Bei der Versuchsreihe Tr-1 konnte bis auf einen Versuch die Belastung nach dem Versagen der Randlamelle bis zum Gesamtversagen gesteigert werden. Das Gesamtversagen dieses Versuchs trat nach dem Versagen der Randlamelle nur unwesentlich unterhalb der zuvor erreichten Traglast ein. In der Versuchsreihe Tr-2 konnte nach dem Versagen der Randlamelle die Traglast bei allen Versuchskörpern gesteigert werden. Nach dem Versagen der Randlamelle konnte dabei ein plastisches Verhalten beobachtet werden. Die Versuchsreihe Tr-3 entsprach der Versuchsreihe Tr-1 mit Ausnahme der KZV. Bei einem Versuch trat nach dem Versagen der Randlamelle ein sofortiges Gesamtversagen ein. Zwei Träger versagten, ohne die bereits zuvor erreichte Traglast erneut zu erreichen. Bei der Versuchsreihe Tr-4 erfolgte bei allen Versuchen eine Traglaststeigerung nach dem Versagen der Randlamelle und ein Gesamtversagen ausgehend von den Holzlamellen oberhalb der zwei FVK-Lamellen. Das Gesamtversagen der meisten Träger der Versuchsreihen Tr-5 und Tr-6 trat nach einem stufigen Versagen, ausgehend von der zweit- bzw. drittuntersten Holzlamelle, auf. Aufgrund des teilweise ausgeprägt plastischen Verhaltens konnte ein Versuch der Reihe Tr-5 und zwei der Reihe Tr-6 nicht bis zum Versagen des Gesamtsystems belastet werden. Das Versagen des Gesamtsystems erfolgte bei der Versuchsreihe Tr-7 nach dem Versagen der untersten Holzlamelle. Das Versagen von zwei der drei Träger der Versuchsreihe Tr-8 erfolgte wie bei der Reihe Tr-7. Beim einem Versuch der Reihe Tr-8 konnte nach einem Anreißen der untersten Holzlamelle und dem damit verbundenen Lastabfall die Last bis zum endgültigen Versagen nochmals gesteigert werden. Eine typische Last-Verformungskurve ist in Bild 7 dargestellt.

In Bild 11 ist das Tragverhalten des Versuchs Tr-2.5 mit einer Randlamelle und des Versuchs Tr-7.4 ohne Randlamelle darstellt. Die dazugehörigen Last-Verschiebungsdiagramme sind in Bild 7 und Bild 9 dargestellt. Beim Versuch Tr-2.5 war zunächst ein Versagen der Randlamelle zu beobachten. Im Anschluss erfolgte ein Ablösen der Randlamelle, bis es zu einem Versagen des Trägers ausgehend von den Holzlamellen oberhalb der zwei FVK-Lamellen kam. Die Biegesteifigkeit nahm dabei nach dem Versagen der Randlamelle kontinuierlich ab. Im Gegensatz hierzu verhielt sich der Prüfkörper Tr-7.4 bis zum Versagen, das ebenfalls von den Holzlamellen oberhalb der FVK-Lamelle ausging, fast bis zur Bruchlast linearelastisch. 
Versuch Tr-2.5

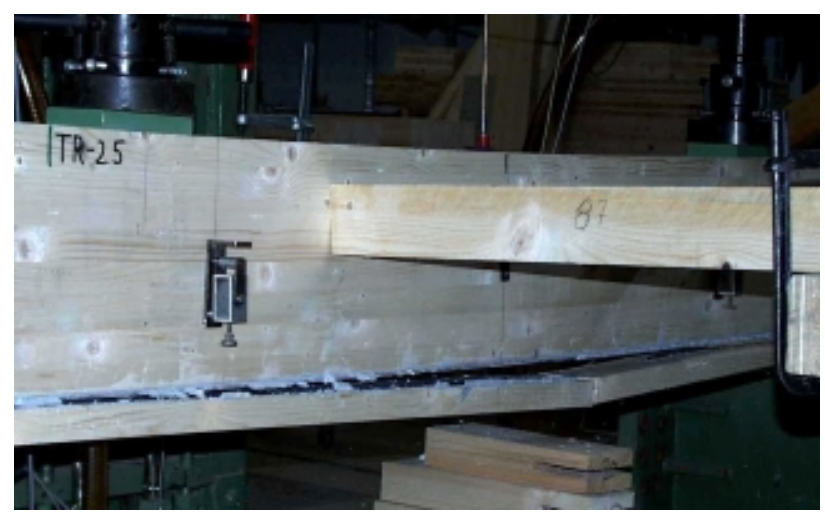

Versagen der Randlamelle und Ablösen

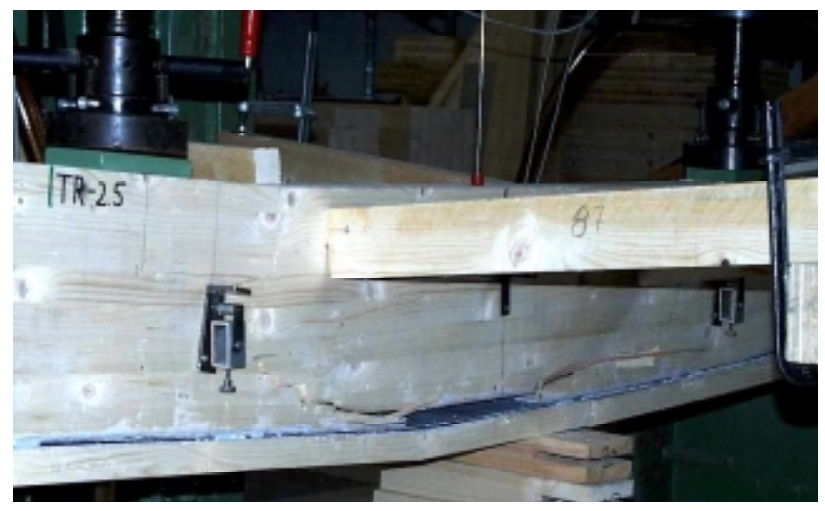

Gesamtversagen ausgehend von der Holzlamelle oberhalb der FVK-Lamelle
Versuch Tr-7.4

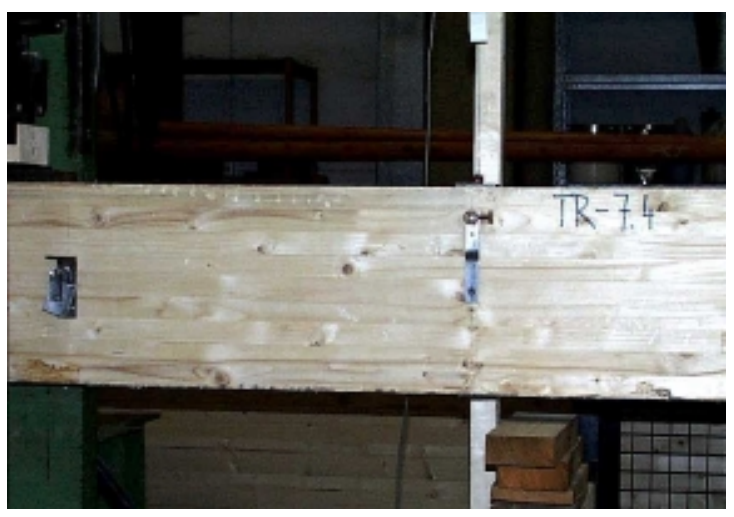

bei ca. $50 \%$ der Traglast

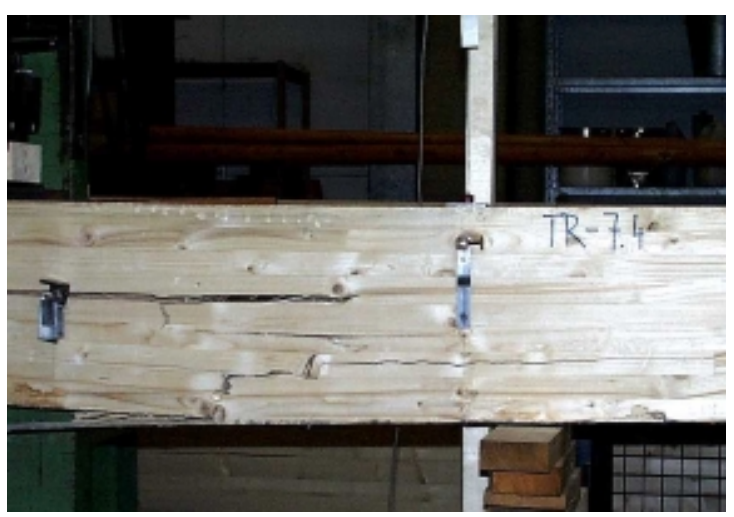

Gesamtversagen ausgehend von der Holzlamelle oberhalb der FVK-Lamelle

Bild 11: Bruchverlauf des Versuchs Tr-2.5 mit Randlamelle (links) und des Versuchs Tr-7.4 ohne Randlamelle (rechts)

Ein Gesamtversagen eines Versuchskörpers aufgrund eines Versagens der FVK-Lamelle erfolgte bei keinem Versuch. Bei Versuchen mit mehreren Lagen von FVK-Lamellen wurden sowohl bei dem Epoxidharzklebstoff, als auch beim Polyurethanklebstoff, keine Ablöseerscheinungen zwischen den einzelnen Lagen der FVK-Lamellen festgestellt. In Bild 12 ist der Ausschnitt des Prüfkörpers Tr-6.1 mit einer ausgeprägten Druckfalte bei versagter Randlamelle dargestellt. Die Randlamelle weist eine geringere Ablösung auf, als die in Bild 11 für den Prüfkörper Tr-2.5 dargestellt wird. 


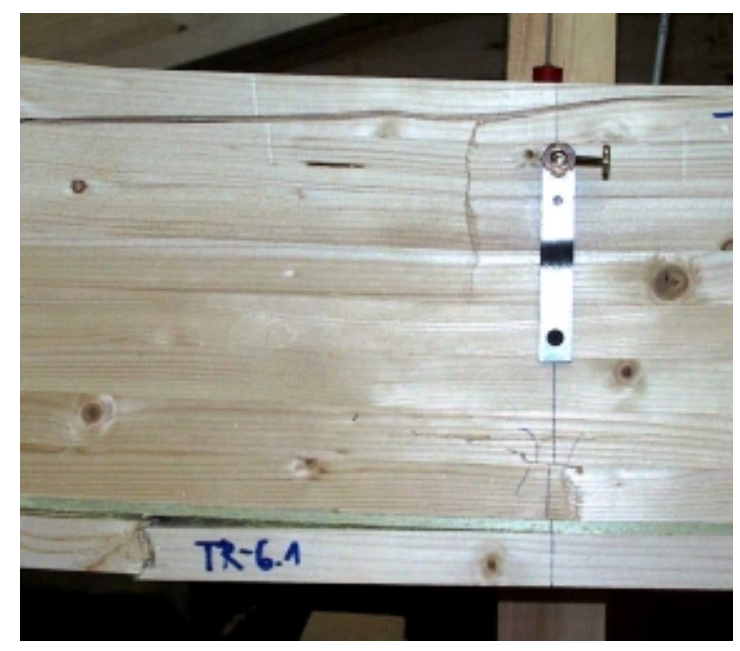

Bild 12: Ausbildung einer Druckfalte und geringes Ablöseverhalten der Randlamelle

Das Versagen des Gesamtsystems wurde teilweise durch ein Herunterdrücken der FVK-Lamelle beim Versagen der oberhalb der FVK-Lamelle liegenden Holzlamellen und einem damit verbundenen Herausziehen der FVK-Lamelle aus dem Auflagerbereich heraus begleitet. In Bild 13 ist das beschriebene Herunterdrücken der FVK-Lamelle dargestellt. Wie ebenfalls in Bild $13 \mathrm{zu}$ sehen, breiteten sich zunächst vertikale Risse meist über zwei bis drei Holzlamellen oberhalb der Verstärkungslage aus, danach in Trägerlängsrichtung.

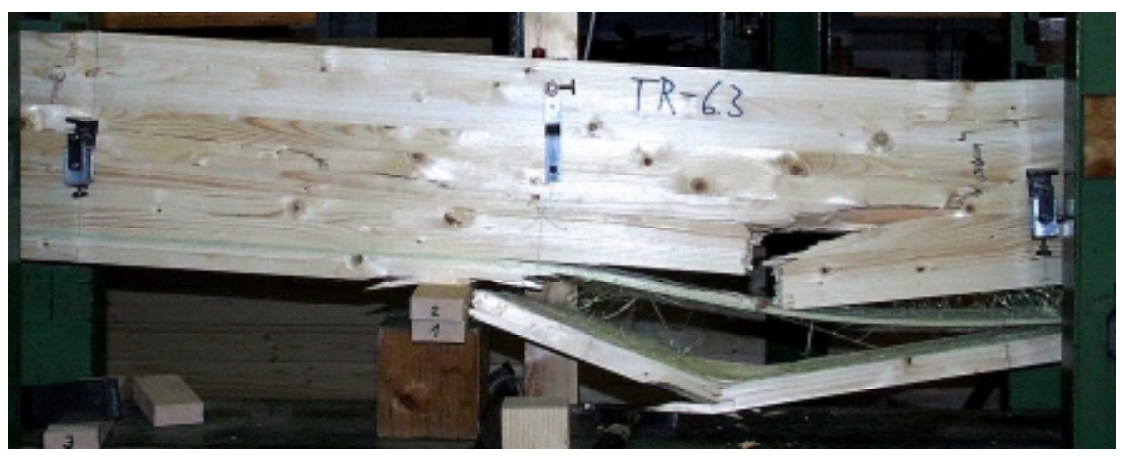

Bild 13: Gesamtversagen des Trägers in Versuch Tr-6.3

\subsubsection{Vergleich der Ergebnisse untereinander}

Aufgrund der fast gleichen Maße der Querschnitte können die einzelnen Versuchsreihen direkt miteinander verglichen werden. Die Einflüsse der Verwendung eines höheren Verstärkungsgrades, von Randlamellen unterhalb der FVK-Lamelle, verschiedenen Klebstoffen und verschiedener FVK-Lamellen sind damit unmittelbar erkennbar. In Tabelle 7 wurden die Mittelwerte der Biegesteifigkeit, der Gesamtverformungen und der Traglasten der einzelnen Versuchsreihen mit den entsprechenden Werten der anderen Versuchsreihen verglichen. 
Tabelle 7: Vergleich der einzelnen Versuchsreihen

\begin{tabular}{|c|c|c|c|}
\hline Vergleich der & $\begin{array}{c}\text { Mittelwert der } \\
\text { Traglast } F_{\max } \\
\mathrm{F}_{\mathrm{a}} / \mathrm{F}_{\mathrm{b}}\end{array}$ & $\begin{array}{c}\text { Mittelwert der } \\
\text { Biegesteifigkeit } \\
\mathrm{El}_{\mathrm{a}} / \mathrm{El}_{\mathrm{b}}\end{array}$ & $\begin{array}{c}\text { Mittelwert der } \\
\text { Verformung } \mathrm{v}_{\max } \\
\mathrm{v}_{\mathrm{a}} / \mathrm{v}_{\mathrm{b}}\end{array}$ \\
\hline Tr-2/Tr-1 & 1,31 & 1,10 & 1,24 \\
\hline Tr-4/Tr-3 & 1,35 & 1,12 & 1,52 \\
\hline Tr-8/Tr-7 & 1,34 & 1,19 & 1,38 \\
\hline Tr-3/Tr-1 & 0,98 & 0,99 & 0,91 \\
\hline Tr-4/Tr-2 & 1,01 & 1,00 & 1,12 \\
\hline Tr-7/Tr-3 & 1,16 & 1,07 & 0,96 \\
\hline Tr-8/Tr-4 & 1,14 & 1,14 & 0,87 \\
\hline Tr-5/Tr-6 & 1,02 & 1,04 & 1,08 \\
\hline
\end{tabular}

Dies zeigt, dass ein höherer Anteil von FVK-Lamellen mit höherem Elastizitätsmodul zu einer deutlicheren Erhöhung der Tragfähigkeit führt als der Biegesteifigkeit.

Den Einfluss des Verstärkungsgrades und eines höheren E-Moduls der FVKLamelle zeigen die Vergleiche zwischen den Versuchsreihen Tr-2 mit Tr-1, Tr-4 mit Tr-3 und Tr-8 mit Tr-7. Durch die Verwendung eines höheren Verstärkungsgrades und eines höheren E-Moduls der CFK-Lamellen wurde die Traglast um 31\%, 35\% und 34\% im Vergleich zur Verstärkung mit einem niedrigeren Verstärkungsgrad und E-Modul erhöht. Gleichzeitig war die Biegesteifigkeit um 10\%, 12\% und 19\% größer.

Aus Tabelle 7 ist zu erkennen, dass ein Einfluss der KZV-Verbindung zwischen den Versuchsreihen Tr-1 und Tr-3 bzw. Tr-2 und Tr-4 auf die Traglast und die Biegesteifigkeit nicht zu erkennen war. Dies kann mit den unterschiedlichen Lamellen im Randbereich erklärt werden. Bei der Versuchsreihe Tr-1 bzw. Tr-2 wurden in den Randbereichen Bretter der Sortierklasse MS7 verwendet, bei der Versuchsreihe Tr-3 bzw. Tr-4 MS10. Der Vergleich zwischen der Reihe Tr-7 und Tr-3 zeigt, dass sich durch die veränderte Lage der Verstärkung um $35 \mathrm{~mm}$ nach unten eine Traglaststeigerung von 16\% und eine Erhöhung der Biegesteifigkeit der Bauteile um $7 \%$ einstellte. Analog ergibt sich für einen Vergleich der Bauteilreihen Tr-8 mit Tr-4 eine Erhöhung der Traglast und der Biegesteifigkeit von jeweils 14\%.

Bei den Versuchsreihen Tr-5 und Tr-6 wurde der Einfluss der Lage der aus MS17 Brettern hergestellten Lamellen als Randlamellen (Reihe Tr-5) bzw. als zweit unterste Lamellen (Reihe Tr-6) untersucht. Vergleicht man die Ergebnisse der Versuchsreihen Tr-5 und Tr-6, so ergibt sich eine Zunahme der Traglast um 2\%, eine Erhöhung der Biegesteifigkeit um 4\% und eine Verformungszunahme um 8\%. Auffallend ist hier die höhere Traglast der Reihe Tr-5 gegenüber Tr-6, da im Gegensatz zur Reihe Tr-5 bei der Reihe Tr-6 oberhalb der AFK-Lamellen eine Lamelle aus MS17 Brettern angeordnet war. Im Gegensatz zu den Versuchen mit CFK-Lamellen 
und Randlamelle erfolgte bei diesen Versuchsreihen das Ablösen der Randlamelle weniger ausgeprägt. Bei einigen Versuchen kam es sogar zu einem Zweitversagen an einer anderen Stelle innerhalb einer Lamelle. Im Versagensbereich wurden dennoch Ablöseerscheinungen festgestellt, die unterschiedlich stark, allerdings nie das Ausmaß der Ablöseerscheinungen der CFK-Lamellen, aufwiesen.

\section{Zusammenfassung}

Die Ergebnisse der Untersuchung zeigen, dass eine Erhöhung der Biegesteifigkeit und der Tragfähigkeit durch das Verstärken von BS-Holz Trägern mit FVK-Lamellen in der Zugzone der Querschnitte erfolgen kann. Die Erhöhung der Tragfähigkeit ist ausgeprägter als die Erhöhung der Biegesteifigkeit durch das Verstärken der Zugzone eines Biegeträgers. Eine Berechnung des E-Moduls nach der Verbundtheorie ergab eine gute Übereinstimmung mit den in Versuchen ermittelten Werten. Die Erhöhung ist sowohl für die Tragfähigkeit, als auch für die Biegesteifigkeit, vom Anteil der Verstärkungsmenge am Gesamtquerschnitt, den Materialeigenschaften der FVK-Lamellen und des Holzes und der Lage der FVKLamellen im Querschnitt abhängig.

Bei Versuchsreihen mit einer Randlamelle aus Holz unterhalb der FVK-Lamelle wurde bei den Versuchsreihen mit CFK-Lamellen ein starkes Ablösen der FVKLamellen nach dem Versagen der Randlamelle festgestellt. Dieser Effekt reduziert die Tragfähigkeit in dem abgelösten Bereich. Bei den Versuchsreihen mit der AFKLamelle wurde ein derart ausgeprägtes Ablösen der Randlamelle nicht beobachtet. Dies hatte bei einigen Versuchen dieser Versuchsreihen zur Folge, dass es in der selben Brettlamelle, an verschiedenen Stellen in Trägerlängsrichtung, zu einem Versagen der jeweiligen Brettlamelle kam. Dies zeigte sich auch durch einen stufigeren Verlauf der Last-Verformungsdiagramme. Bei einer Reduzierung des Ablöseeffektes sind zusätzliche Steigerungen der Tragfähigkeit zu erwarten.

Bei Klebefugen zwischen den einzelnen FVK-Lamellen konnten weder beim Epoxidharzklebstoff noch beim Polyurethanklebstoff ein Versagen festgestellt werden. Ein Versagen der Klebefuge konnte nur zwischen der Holz - FVK-Lamelle, ausgelöst durch das Gesamtversagen bzw. das bereits beschriebene Ablösen, festgestellt werden. Bei einigen der Versuchsreihen mit einem hohen Verstärkungsgrad konnten sichtbare Druckfalten bis zu 1/3 der Querschnittshöhe beobachtet werden.

Das Verstärken von BS-Holz Trägern ist mit im Holzbau gängigen Klebstoffen und Herstellungsverfahren möglich und bietet die Möglichkeit, signifikante Steigerungen der Tragfähigkeit und Biegesteifigkeit zu erzielen.

\section{Literatur}

Biblis EJ (1965) Analysis of wood-fiberglass beams within and beyond the elastic region. Forest Prod. 25 (224) 1965: 81-88

Blaß HJ, Romani M (2000) Trag- und Verformungsverhalten von Verbundträgern aus Brettschichtholz und faserverstärkten Kunststoffen. Forschungsbericht der 
Versuchsanstalt für Stahl, Holz und Steine, Abt. Ingenieurholzbau der Universität Karlsruhe

Bulleit WM, Sandberg LB, Woods GJ (1989) Steel-reinforced glued laminated timber; J. Structural Engineering 115(2): 433-444

Ehlbeck J, Colling F (1987) Tragfähigkeit von Glasfaser-Verbund-Profilen verstärkten Brettschichtholzträgern. Forschungsbericht der Versuchsanstalt für Stahl, Holz und Steine, Abt. Ingenieurholzbau der Universität Karlsruhe

Hernandez R, Davalos JF, Sonti SS, Kim Y, Moody RC (1997) Strength and stiffness of reinforced Yellow-Poplar glued-laminated beams; Res. Pap. FPL-RP-554. Madison, WI: U.S. Department of Agriculture, Forest Service, Forest Products Laboratory

Lantos GG (1970) The flexural behaviour of steel reinforced laminated timber beams. Canadian Wood Council Wood Science. 2(3):136-143

Rowlands RE, Van Deweghe RP, Laufenberg TL, Krueger GP (1986) Fiberreinforced wood composites. Wood and Fiber Science, 18(1), 1986: 39-57.

Peterson J (1965) Wood beams prestressed with bonded tension elements. Journal of the Structural Division, STI: 103-119

Plevris N, Triantafillou TC (1992) FRP-reinforced wood as structural material. American Society of Civil Engineers. Journal of Materials in Civil Engineering. 4(3): 300-317

Triantafillou TC, Deskovic N (1992) Prestressed FRP sheets as external reinforcement of wood members; J. Struct. Eng., ASCE, 118(5), 1270

Van de Kuilen J-WG (1991). Theoretical and experimental research on glass fibre reinforced laminated timber beams. 1991 International timber engineering conference, London 3.226-3.231 